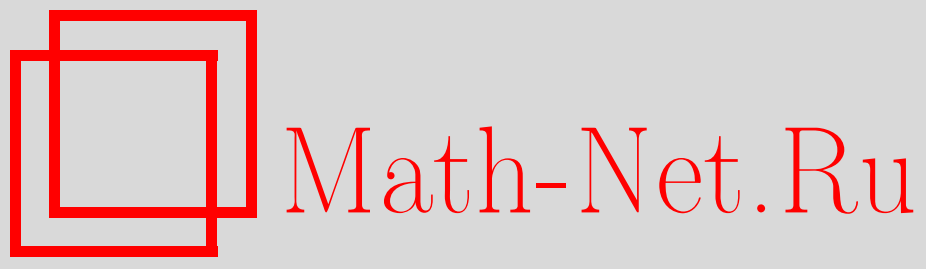

С. В. Асташкин, Об интерполяции пересечений, порожденных линейным функционалом, Функи. анализ и его прил., 2005, том 39, выпуск 2, 61-64

DOI: https://doi.org/10.4213/faa40

Использование Общероссийского математического портала MathNet.Ru подразумевает, что вы прочитали и согласны с пользовательским соглашением

http://www . mathnet.ru/rus/agreement

Параметры загрузки:

IP: 3.85 .5 .30

26 апреля 2023 г., 14:11:47

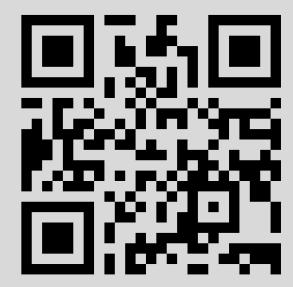




\title{
Об интерполяции пересечений, порожденных линейным функционалом
}

\author{
(c) 2005. С. В. АСТАшкИН
}

1. Введение. Пусть $\left(X_{0}, X_{1}\right)$ - банахова пара, т. е. два банаховых пространства, линейно и непрерывно вложенных в отделимое линейное топологическое пространство $\mathscr{T}$. Если $N$ - линейное подпространство в $\mathscr{T}$, то оно порождает нормированную (вообще говоря, не банахову) пару $\left(X_{0} \cap N, X_{1} \cap N\right)$, где норма в $X_{i} \cap N$ - сужение нормы пространства $X_{i}(i=0,1)$.

Проблема интерполяции пересечений состоит в нахождении условий на тройку $\left(X_{0}, X_{1}, N\right)$ и параметры вещественного метода интерполяции $\theta \in(0,1)$ и $q \in[1, \infty]$, при которых верно следующее естественное равенство (по составу элементов с эквивалентностью норм):

$$
\left(X_{0} \cap N, X_{1} \cap N\right)_{\theta, q}=\left(X_{0}, X_{1}\right)_{\theta, q} \cap N
$$

(определение пространств $\left(X_{0}, X_{1}\right)_{\theta, q}$ см. ниже).

Важный частный случай этой задачи, когда $\left(X_{0}, X_{1}\right)$ - пара весовых $L_{p}$-пространств $\left(L_{p}\left(w_{0}\right), L_{p}\left(w_{1}\right)\right)$, а $N$ - линейное пространство всех функций $f:(0, \infty) \rightarrow \mathbb{R}$, таких, что

$$
\int_{0}^{\infty} f(x) d x=0
$$

был рассмотрен в работе Кругляка, Малигранды и Перссона [1]. Там же была поставлена более общая задача: при каких условиях на $w_{0}(x), w_{1}(x), p_{0}, p_{1} \in$ $[1, \infty), \theta \in(0,1)$ и $q \in[1, \infty)$

$$
\left(L_{p_{0}}\left(w_{0}\right) \cap N, L_{p_{1}}\left(w_{1}\right) \cap N\right)_{\theta, q}=\left(L_{p_{0}}\left(w_{0}\right), L_{p_{1}}\left(w_{1}\right)\right)_{\theta, q} \cap N
$$

(здесь, вообще говоря, $p_{0} \neq p_{1}$, а пространство $N$ определяется по-прежнему соотношением (2))? Она и стала отправной точкой для постановки проблемы, рассматриваемой в данной заметке.

Предположим, что $N$ - ядро линейного функционала $\psi$, определенного на некотором линейном пространстве $M \subset \mathscr{T}, \psi \in\left(X_{0} \cap X_{1}\right)^{*}$. Мы определим четыре индекса растяжения $\mathscr{K}$-функционала $\psi$ в сопряженной паре $\left(X_{0}^{*}, X_{1}^{*}\right)$, используя которые сформулируем необходимые условия для выполнения равенства (1). При подходящем выборе $N$ эти условия оказываются также и достаточными. В качестве следствия мы получаем результаты Иванова и Калтона [2] о сравнении интерполяционных пространств $\left(X_{0}, X_{1}\right)_{\theta, q}$ и $\left(N_{0}, X_{1}\right)_{\theta, q}$, где $\psi \in X_{0}^{*}$ и $N_{0}=\operatorname{Ker} \psi$ с нормой из $X_{0}$. В то же время доказательство теоремы 1 основано на использовании идеи из последней работы, состоящей в сведении общей задачи интерполяции подпространств к изучению оператора сдвига в некотором весовом $l_{p}$-пространстве последовательностей. При определенных условиях полученные результаты позволяют решить задачу об интерполяции пересечений весовых $L_{p}$-пространств, поставленную в [1] (см. равенство (3)). Об этом речь пойдет в конце работы.

В заключение заметим, что задача об интерполяции пересечений, а также задача, рассматриваемая в [2], - важные (но все-таки частные) случаи общей 
и трудной проблемы интерполяции подпространств, сформулированной еще в монографии Лионса и Мадженеса [3] (см., в частности, [4-13]).

2. Определения и обозначения. Для нормированной пары $\left(X_{0}, X_{1}\right)$ и $t>0 \mathscr{K}$ - и $\mathscr{J}$-функционалы Петре определяются следующим образом:

$$
\begin{array}{lll}
\mathscr{K}\left(t, x ; X_{0}, X_{1}\right)=\inf _{x=x_{0}+x_{1}, x_{i} \in X_{i}}\left(\left\|x_{0}\right\|_{X_{0}}+t\left\|x_{1}\right\|_{X_{1}}\right), & & x \in X_{0}+X_{1}, \\
\mathscr{J}\left(t, x ; X_{0}, X_{1}\right)=\max \left(\|x\|_{X_{0}}, t\|x\|_{X_{1}}\right), & & x \in X_{0} \cap X_{1} .
\end{array}
$$

Если $0<\theta<1$ и $1 \leqslant q<\infty$, то интерполяционное пространство вещественного метода $X_{\theta, q}=\left(X_{0}, X_{1}\right)_{\theta, q}$ состоит из всех $x \in X_{0}+X_{1}$, таких, что

$$
\|x\|_{X_{\theta, q}}=\left\{\int_{0}^{\infty}\left(t^{-\theta} \mathscr{K}\left(t, x: X_{0}, X_{1}\right)\right)^{q} \frac{d t}{t}\right\}^{1 / q}<\infty .
$$

Если $X_{0} \cap X_{1}$ всюду плотно в $X_{0}$ и в $X_{1}$, а $\psi \in\left(X_{0} \cap X_{1}\right)^{*}$, то можно рассмотреть банахову пару сопряженных пространств $\left(X_{0}^{*}, X_{1}^{*}\right)$ и $\psi \in X_{0}^{*}+X_{1}^{*}[14, \S 3.7]$. Важную роль в дальнейшем играет $\mathscr{K}$-функционал $k(t)=\mathscr{K}\left(t, \psi ; X_{0}^{*}, X_{1}^{*}\right)$, а также функции

$$
M(t)=\sup _{s>0} \frac{k(t s)}{k(s)}, \quad M_{0}(t)=\sup _{0<s \leqslant \min (1,1 / t)} \frac{k(t s)}{k(s)}, \quad M_{\infty}(t)=\sup _{s \geqslant \max (1,1 / t)} \frac{k(t s)}{k(s)} .
$$

Они полумультипликативны при $t>0$, и поэтому существуют числа

$$
\begin{aligned}
\alpha & =\lim _{t \rightarrow 0} \frac{\log _{2} M(t)}{\log _{2} t}, & \beta & =\lim _{t \rightarrow \infty} \frac{\log _{2} M(t)}{\log _{2} t}, \\
\alpha_{0} & =\lim _{t \rightarrow 0} \frac{\log _{2} M_{0}(t)}{\log _{2} t}, & \beta_{\infty} & =\lim _{t \rightarrow \infty} \frac{\log _{2} M_{\infty}(t)}{\log _{2} t},
\end{aligned}
$$

называемые индексами растяжения функции $k(t)$. Легко видеть, что $0 \leqslant \alpha \leqslant$ $\alpha_{0} \leqslant \beta \leqslant 1$ и $0 \leqslant \alpha \leqslant \beta_{\infty} \leqslant \beta \leqslant 1$. Если, в частности, $\psi \in X_{0}^{*}$, то $k(t) \leqslant C$ $(t>0)$. Поэтому $\alpha=\beta_{\infty}=0$. Оставшиеся два индекса $\alpha_{0}$ и $\beta$ были введены и использовались в работе [2].

3. Основные результаты. Предположим, что $\left(X_{0}, X_{1}\right)$ - банахова пара, такая, что $X_{0} \cap X_{1}$ всюду плотно в $X_{0}$ и в $X_{1}$. Пусть $\psi \in\left(X_{0} \cap X_{1}\right)^{*}, \psi \neq 0$, $N=\operatorname{Ker} \psi$ и $N_{i}$ - пространство $N$, рассматриваемое с нормой из $X_{i}(i=0,1)$.

TEOPEMA 1. Eсли

$$
\theta \in(0, \alpha) \cup\left(\beta_{\infty}, \alpha_{0}\right) \cup(\beta, 1),
$$

то нормы интерполячионных пространств $N_{\theta, q}=\left(N_{0}, N_{1}\right)_{\theta, q}$ u $X_{\theta, q}=\left(X_{0}, X_{1}\right)_{\theta, q}$ эквивалентны на $N$. При условии $\beta_{\infty} \leqslant \alpha_{0}$ верно и обратное утвержсение: если нормы пространств $N_{\theta, q}$ и $X_{\theta, q}$ эквивалентны на $N$, то выполнено (4).

Кроме того, если $\theta \in(0, \alpha) \cup(\beta, 1)$, то $N_{\theta, q}$ всюду плотно в $X_{\theta, q} ;$ если $\theta \in\left(\beta_{\infty}, \alpha_{0}\right)$, то $N_{\theta, q}$ всюду плотно в некотором подпространстве коразмерности 1 пространства $X_{\theta, q}$.

СлЕдСтвиЕ 1. Предположим, что линейный функиионал $\psi$ определен на некотором линейном пространстве $M \supset X_{0} \cap X_{1}, N=\operatorname{Ker} \psi$. Пусть $\psi_{0}-$ сужсение функиионала $\psi$ на $X_{0} \cap X_{1}, \psi_{0} \in\left(X_{0} \cap X_{1}\right)^{*}, \psi_{0} \neq 0, u \alpha, \beta, \alpha_{0} u$ $\beta_{\infty}-$ индексы растяэсения функиии $k(t)=\mathscr{K}\left(t, \psi_{0} ; X_{0}^{*}, X_{1}^{*}\right), \beta_{\infty} \leqslant \alpha_{0}$. Тогда, если верно равенство (1), то выполнено (4). 
Tеорема 2. Пусть $\psi_{0} \in\left(X_{0} \cap X_{1}\right)^{*}, \psi_{0} \neq 0$. Eсли $\theta \in\left(\beta_{\infty}, \alpha_{0}\right)$, mo $\psi_{0}$ продолэсается по непрерьвности до функиионала $\psi \in\left(\left(X_{0}, X_{1}\right)_{\theta, q}\right)^{*}$. Если $\theta \in$ $(0, \alpha)$ (соответственно $\theta \in(\beta, 1))$, то $\psi_{0}$ продолэсается по непрерьвности до бункционала $\psi \in\left(\left(X_{0}, X_{1}\right)_{\theta, q} \cap X_{1}\right)^{*}\left(\right.$ соответственно $\left.\psi \in\left(\left(X_{0}, X_{1}\right)_{\theta, q} \cap X_{0}\right)^{*}\right)$. Кроме того, если $N=\operatorname{Ker} \psi$, то равенство (1) будет верным во всех трех случаях.

Из теорем 1 и 2 получаем важное

СлЕДСтвиЕ 2. Пусть $\psi \in\left(X_{0} \cap X_{1}\right)^{*}, \psi \neq 0, N=\operatorname{Ker} \psi$. Если $\theta \in(0, \alpha) \cup$ $(\beta, 1)$, то интерполящионное пространство $X_{\theta, q}=\left(X_{0}, X_{1}\right)_{\theta, q}$ совпадает $c$ множеством всех $x \in X_{0}+X_{1}$, таких, что $x=\sum_{k=-\infty}^{\infty} x_{k}, x_{k} \in N, u$

$$
\left(\sum_{k=-\infty}^{\infty}\left(\mathscr{J}\left(2^{k}, x_{k} ; X_{0}, X_{1}\right) 2^{-\theta k}\right)^{q}\right)^{1 / q} \leqslant C\|x\|_{X_{\theta, q}}
$$

где константа $C>0$ не зависит от $x$. Аналогичное утверждение верно также в случае $\theta \in\left(\beta_{\infty}, \alpha_{0}\right)$, если пространство $X_{\theta, q}$ заменить пересечением $X_{\theta, q} \cap \tilde{N}$, где $\tilde{N}$ - ядро функиионала $\tilde{\psi}$, являющегося продолжением функционала $\psi$ на $X_{\theta, q}$.

Отсюда легко получить результаты о сравнении пространств $\left(X_{0}, X_{1}\right)_{\theta, q}$ и $\left(N_{0}, X_{1}\right)_{\theta, q}$, где $N_{0}=\operatorname{Ker} \psi, \psi \in X_{0}^{*}$, из работы [2]. Действительно, в этом случае $\alpha=\beta_{\infty}=0$. Кроме того, так как $\psi$ определен на всем пространстве $X_{0}$, то $\left(X_{0}, X_{1}\right)_{\theta, q} \subset N_{0}+X_{1}$. Поэтому ввиду следствия 2 и теоремы о совпадении пространств $\mathscr{K}$ - и $\mathscr{J}$-методов [14, теорема 3.3.1]

$$
\left(N_{0}, X_{1}\right)_{\theta, q}=\left(X_{0}, X_{1}\right)_{\theta, q}, \quad \text { если } \theta \in(\beta, 1),
$$

и

$$
\left(N_{0}, X_{1}\right)_{\theta, q}=\left(X_{0}, X_{1}\right)_{\theta, q} \cap \tilde{N}, \quad \text { если } \theta \in\left(0, \alpha_{0}\right) .
$$

Дополнительно из теоремы 1 следует, что $\left(N_{0}, X_{1}\right)_{\theta, q}$ не замкнуто в $\left(X_{0}, X_{1}\right)_{\theta, q}$, если $\theta \in\left[\alpha_{0}, \beta\right]$.

4. Приложения. Вернемся к задаче, связанной с равенством (3). В этом случае пространство $N$, определяемое соотношением (2), является ядром функционала $\psi(f)=\int_{0}^{\infty} f(x) d x$, область определения $M$ которого состоит из всех измеримых функций $f:(0, \infty) \rightarrow \mathbb{R}$, таких, что $\int_{a}^{b}|f(x)| d x<\infty$ для произвольных $0<a<b<\infty$, а также существует $\lim _{a \rightarrow 0, b \rightarrow \infty} \int_{a}^{b} f(x) d x$.

Для простоты мы ограничимся случаем, когда $1 \leqslant p_{0}<p_{1}<\infty$, а также выполнены условия регулярности, аналогичные условиям из работы [1]. А именно, предположим, что

(*) $w_{0}(x)$ возрастает, $w_{0}(2 x) \leqslant C_{1} w_{0}(x)(x>0)$ и $\int_{x}^{\infty} w_{0}(s)^{1 /\left(1-p_{0}\right)} d s \leqslant$ $C_{2} x w_{0}(x)^{1 /\left(1-p_{0}\right)}(x>0)$, если $p_{0}>1$, а $w_{1}(x)$ либо убывает и $w_{1}(x) \leqslant C_{3} w_{1}(2 x)$ $(x>0)$, либо возрастает, но так, что функция $w(x)=w_{0}(x) / w_{1}(x)$ возрастает и $\int_{0}^{x} w_{1}(s)^{1 /\left(1-p_{1}\right)} d s \leqslant C_{4} x w_{1}(x)^{1 /\left(1-p_{1}\right)}(x>0)$.

Пусть $r^{-1}=p_{0}^{-1}-p_{1}^{-1}, v(x)=w_{0}(x)^{p_{1} /\left(p_{1}-p_{0}\right)} w_{1}(x)^{-p_{0} /\left(p_{1}-p_{0}\right)}$ и $\gamma(t)-$ peшение уравнения $\int_{0}^{x} v(s) d s=t^{-r}$ для каждого $t>0$.

Теорема 3. Пусть $p_{0}, p_{1}, w_{0}(x)$ и $w_{1}(x)$ удовлетворяют условиям $(*), a \alpha$, $\beta, \alpha_{0} u \beta_{\infty}-$ индексы растяэсения функиии $k(t)=\gamma(t)^{\left(p_{0}-1\right) / p_{0}} w_{0}(\gamma(t))^{-1 / p_{0}}$, 
$\beta_{\infty} \leqslant \alpha_{0}$. Тогда равенство (3) верно в том и только том случае, когда выполнено (4).

В частности, рассмотрим случай степенных весовых функций $w_{0}(x)=x^{l}$ и $w_{1}(x)=x^{m}$. Тогда условия $(*)$ выполняются, если (а) $l>p_{0}-1$ и $m \leqslant 0$ или (b) $l>p_{0}-1, l>m$ и $0 \leqslant m<p_{1}-1$. Так как $k(t) \asymp t^{\theta_{0}}$, где $\theta_{0}=\frac{p_{1}\left(l-p_{0}+1\right)}{p_{1}(l+1)-p_{0}(m+1)}$, то $\alpha=\beta=\alpha_{0}=\beta_{\infty}=\theta_{0}$, и мы получаем

СлеДСТВие 3. Предположим, что для $p_{0}, p_{1}\left(1 \leqslant p_{0}<p_{1}<\infty\right), l$ u выполнено хотя бы одно из условий (a) и (b). Тогда равенство

$$
\left(L_{p_{0}}\left(x^{l}\right) \cap N, L_{p_{1}}\left(x^{m}\right) \cap N\right)_{\theta, q}=\left(L_{p_{0}}\left(x^{l}\right), L_{p_{1}}\left(x^{m}\right)\right)_{\theta, q} \cap N
$$

выполнено в том и только том случае, когда $\theta \neq \frac{p_{1}\left(l-p_{0}+1\right)}{p_{1}(l+1)-p_{0}(m+1)}$.

В случае $p_{0}=p_{1}=q$ последнее утверждение иным способом было доказано в $[1$, Corollary 1$]$.

Автор благодарен проф. Л. Малигранда за полезные обсуждения некоторых вопросов, связанных с доказательством теоремы 2.

\title{
ЛитератУра
}

1. Krugljak N., Maligranda L., Persson L.-E. Ark. Mat., 37, 323-344 (1999). 2. Ivanov S., Kalton N. Алгебра и анализ, 13, No. 2, 93-115 (2001). 3. Лионс Ж. Л., Мадженес Э. Неоднородные граничные задачи и их приложения. Мир, М., 1971. 4. Triebel H. Math. Nachr., 69, 57-60 (1975). 5. Трибель X. Теория интерполяции, функциональные пространства, дифференциальные операторы. Мир, М., 1980. 6. Maligranda L. Suppl. Rend. Circ. Mat. Palermo, 10, 113-118 (1985). 7. Wallsten R. In: Lect. Notes in Math., Vol. 1902, 1988, pp. 410-419. 8. Pisier G. Pasific J. Math., 155, 341-368 (1992). 9. Janson S. Ark. Math., 31, 307-338 (1993). 10. Кисляков С. В., Куанхуа Шу. Алгебра и анализ, 8, вып. 4, 75-109 (1996). 11. Löfström J. Interpolation of subspaces, Technical report No. 10, Univ. of Göteborg, 1997. 12. Astashkin S. V. J. Math. Math. Sci., 25, No. 7, 451-465 (2001). 13. Kaijser S., Sunehag P. Interpolation subspaces and the unit problem, In: Function Spaces, Interpolation Theory and Related Topics (Lund 2000), de Gruyter, Berlin, 2002, pp. 345-353. 14. Берг Й., Лефстрем Й. Интерполяционные пространства. Введение. Мир, М., 1980.

Самарский госуниверситет

e-mail: astashkn@ssu.samara.ru

Поступило в редакцию 28 августа 2003 г.

УДК 517.44

\section{Модифицированный двоичный интеграл и производная дробного порядка на $\mathbb{R}_{+}{ }^{*}$}

\author{
(с) 2005. Б. И. Голуьов
}

1. В работе [1] Бутцер и Вагнер ввели сильную двоичную производную и интеграл порядка $r=1,2, \ldots$ и доказали, что они являются взаимно обратными операторами. Их определения относятся к функциям $f \in L[0,1)$ или $f \in L(G)$, где $G$ - двоичная группа, изоморфная модифицированному отрезку $[0,1]^{*}$.

*Работа поддержана РФФИ, грант 05-01-00206. 